Proceedings of SALT 22: 307-325, 2012

\title{
A unified analysis of the same, phrasal comparatives, and superlatives *
}

\author{
Carl Pollard \\ The Ohio State University
}

\author{
E. Allyn Smith \\ University of Quebec at Montreal
}

\begin{abstract}
We present a unified categorial analysis of several types of English comparative, superlative, and the sameldifferent (S/D) sentences, thereby accounting for parallels among these constructions first noted in Heim ms. Our analysis, couched in a linear-logic-based form of categorial grammar along the lines of Oehrle 1994, builds on the basic insight underlying Barker's (2007) 'parasitic scope' analysis of internal readings of the same, but is simpler and more general than Barker's. Ours is also the first unified analysis of all three kinds of phenomena. Our analysis of phrasal comparatives captures their essential similarity to associate-remnant S/D constructions such as Anna read the same book as Bill.
\end{abstract}

Keywords: parasitic scope, phrasal comparatives, superlatives, same/different

\section{Introduction}

This paper has two interrelated purposes. The first is to analyze similarities between the syntax and semantics of English comparative and superlative constructions on the one hand and the same/different (hereafter, S/D) constructions on the other, some of which have been noted in the linguistic literature starting with Heim ms. In particular, we give the first unified formal analysis of these three kinds of phenomena that captures their similarities. The second goal is to highlight a categorial framework, or, more precisely, a family of closely related frameworks, for syntactic and semantic analysis, based on linear logic, which has attracted considerable attention in recent years among researchers interested in interconnections among logic, language, and computation, but which deserve more attention from linguists concerned with language description and analysis. More specifically, we will argue that certain architectural properties of the framework enable it to provide simple, unified, and elegant analyses of these difficult constructions, namely: (1) distinguishing between the linear-logic-based tectogrammar, which drives the argument structure and, therefore, the semantic composition, and phenogrammar, which determines surface

* We thank the SALT abstract referees as well as the conference audience for their comments, questions, and suggestions.

C2012 Pollard and Smith 
word order; and (2) the robust expressive power of the higher-order-logic based phenogrammar, which allows for succinct and precise characterizations of string operations such as deletion, extraposition, and lowering.

The paper is organized as follows. In the following section, we present some English data which exemplify the parallels between comparatives/superlatives and $\mathrm{S} / \mathrm{D}$, identifying the subset of this data that we will be concerned with here. Section 3 sketches the categorial framework, here called linear grammar (LG) due to the fact that its tectogrammar is a linear logic, which will be employed for the linguistic analysis. Section 4 presents detailed analyses of representative sentences. Finally, section 5 summarizes and concludes the paper. (Unfortunately, considerations of space preclude detailed comparison with competing analyses.)

\section{Data}

Superlatives and comparatives on the one hand, and the same and different on the other hand, exhibit remarkable parallelism in terms of the multiplicity of types of constructions in which they can appear. Here we note two of those construction types that are the main focus of this paper. ${ }^{1}$

\subsection{External vs. Internal Readings}

Both superlatives and S/D exhibit an ambiguity between an internal reading, which depends on the presence of an expression called the associate (or, in Heim's terminology, the correlate), and the external reading, also called an absolute or anaphoric reading, which does not. Each is explained further below.

In external readings, such as those in (1), the superlative or S/D expression depends for its interpretation on a presupposed entity or set of entities retrievable from the utterance context. Contexts are indicated in parentheses in our examples. In the case of external superlatives, what is presupposed is the set over which the quantity in question (in (1a), the degree of meanness) is maximized (here, the set of weird scary dogs in our neighborhood). In the case of S/D constructions, on the other hand, what is presupposed is the entity with respect to which (non-)identity is determined (in (1b-c), the book Goodnight Moon).

(1) a. (There are lots of weird scary dogs in our neighborhood.) The meanest dog bit Felix.

1 Note, however, that there are others, e.g. clausal constructions (or comparative sub-deletion) such as Kim bought more books than/the same book as/a different book than Sandy sold. For reasons of space, we have chosen to focus on the two most difficult constructions for our analysis, both of which require handling ambiguities not present with these clausal examples. 
'The same', phrasal comparatives and superlatives

b. (Chris and Dana read Goodnight Moon.) Kim and Sandy read the same book.

c. (Chris and Dana read Goodnight Moon.) But Kim and Sandy read a different book/different books.

The key thing to note here is that the interpretation of the superlative or S/D expression is independent of, or external to, the utterance in which it occurs. By contrast, in the case of internal readings such as those exemplified in (2), the interpretation of the superlative or S/D expression depends on the presence of a focused expression-the associate - in the same utterance. More specifically, as we will discuss in section 4, adapting and generalizing Barker's (2007) 'parasitic scope' analysis of internal $\mathrm{S} / \mathrm{D}$ readings, the superlative or S/D expression takes as semantic arguments both (i) the meaning of the associate (indicated in examples by bolding), and (ii) the continuation, which, roughly speaking -we will make this more precise in due course - is the functional abstraction of the local context consisting of the entire utterance with two 'holes' corresponding to the associate and the superlative or S/D expression respectively. ${ }^{2}$

(2) a. (There's been a big problem in our neighborhood of cats getting bitten by dogs.) The meanest dog bit Felix.

b. Kim and Sandy read the same book.

c. Kim and Sandy read different books.

In (2a), the interpretation can be described informally as the proposition that the meaning of the associate, namely Felix, (uniquely) maximizes the function $f$ that maps cats in our neighborhood (the contrast set) to the highest degree of meanness possessed by the meanest dog that bit them. In this internal reading, the function $f$ is characterized in terms of the binary relation of biting, which happens to be precisely the continuation consisting of the utterance (2a) with two 'holes' corresponding to the superlative expression the meanest dog and the associate Felix.

In internal readings of the same sentences such as (2b), the associate is a plural (or a generalized quantifier ranging over plurals), in this case Kim and Sandy, and the interpretation is that there is a constant function $f$ from the set of atomic elements of that plural (here, the set whose only members are Kim and Sandy) to the set

2 Although we follow Barker in using the term 'continuation' for expressions which would be analyzed in mainstream generative grammar (hereafter, MGG) as the scope of a 'covertly moved' operator, we do not adopt his technical implementation, in which continuations are treated as having different syntactic types from ordinary categorial functors. The only thing that differentiates our continuations from other functor expressions is that they are never applied to their arguments; instead, they themselves become the arguments of functors which are phenogrammatically 'lowered' into them, Oehrle-style. 
denoted by the complement common noun of the determiner the same (here, the set of books) such that, for each $x$ in the domain of $f$, the ordered pair $\langle x, f(x)\rangle$ is in the relation of reading, which happens to be precisely the continuation consisting of the utterance (2b) with two 'holes' corresponding to the S/D expression the same book and the associate Kim and Sandy. Truth-conditionally, this amounts to the existence of a book which both Kim and Sandy read. Note the parallel here between the analyses of internal readings of superlative sentences with those of S/D sentences: in both cases, a quantifier-like element (the S/D or superlative expression) takes two arguments, namely (i) the associate, and (ii) a two-holed continuation (with one hole corresponding to the associate and the other to the quantifier-like element itself).

Internal readings of different sentences such as (2c) are essentially similar, but with 'constant' replaced by 'injective' in the description of their continuations (and thus, the analysis we present in this paper for same extends to these cases as well). Truth-conditionally, this amounts to there being distinct books that were read by Kim and Sandy respectively.

A further point to be noted in connection with internal readings is that ambiguities can arise with respect to which expression is the associate. ${ }^{3}$

a. i. Jo owes Mo the most. (Jo owes Mo more than anyone else does.)

ii. Jo owes Mo the most. (Jo owes Mo more than s/he owes anyone else.)

b. i. Kim and Sandy gave Mo and Jo the same book. (Kim gave Mo and Jo the same book as Sandy did.)

ii. Kim and Sandy gave Mo and Jo the same book. (Kim and Sandy gave Mo the same book as they gave Jo.)

\subsection{Remnant Constructions}

In the literature on comparatives, a distinction is often drawn between clausal constructions, where the complement of than or as is a clause from which (an expression containing) a degree expression appears to have been (sub-)deleted, and phrasal constructions, where the complement of than or as is an 'intact' phrase (cf. Kennedy 1999). Within the latter, we can also distinguish a subclass of remnant comparatives, such as (4a), where the matrix clause to which the than- or as-phrase attaches contains both the comparative expression (here, more) and the associate

3 Such ambiguities, which are also found in the associate-remnant constructions discussed below, can often be resolved on the basis of pitch accent placement. Unfortunately, accentual disambiguation of these constructions is beyond the scope of this paper. 
'The same', phrasal comparatives and superlatives

(here, Bill), and the comparison drawn is between the meaning of the associate and the meaning of the complement of than or as, which is called the remnant (so-called because on some analyses of such sentences, the complement of than or as begins life as a full clause, and the expression in question is what remains of it after a process of ellipsis has applied to it, in the present case Clara, cf. Bhatt \& Takahashi 2007).

Informally, we can describe the meaning of (4a) as the proposition that the amount Anna owes Bill exceeds the amount she owes Clara. On our analysis of the remnant comparative, as in our analyses of superlatives and S/D constructions, a continuation with two 'holes' plays an important role. The continuation is the matrix clause (i.e. the clause to which the than-phrase attaches) with two 'holes' corresponding to the comparative expression (here, more) and the associate (here, Bill). Thus, the meaning of more can be analyzed as a function that takes three arguments: the continuation (which itself is a binary relation), the meaning of the associate, and the meaning of the remnant. It returns the proposition that there is an inequality between the amount denoted by the application of the continuation to the associate and the amount denoted by the application of that same continuation to the remnant. In other words, here the continuation is Anna owes [someone] [some amount] where brackets indicate the unsatisfied arguments. Then, applying that continuation to the associate gives the meaning that Anna owes Bill [some amount] and applying it to the remnant gives the meaning that Anna owes Clara [some amount] and more asserts that the former quantity is greater than the latter.

Note that, as with internal readings (and numerous other types of focus constructions), there are ambiguities of remnant constructions hinging on which expression is the associate, as in the difference between (4a) and (4b). Our analysis handles these easily via the difference between the associate and the argument that is neither the associate nor the remnant (i.e. the argument that, were it the associate, would give rise to the other reading) in terms of their role in our analysis. While the associate is a separate argument of more, the non-associate non-remnant argument is part of the continuation. Essentially, more applies the meaning of the continuation twice in the construction of the propositional meaning (once to the associate and once to the remnant), accounting for why the paraphrases of these sentences' meanings include a double mention of the name of the non-associate non-remnant argument. So, whereas the continuation in (4a) was Anna owes [someone] [some amount], the continuation in (4b) is [Someone] owes Bill [some amount].

(4) a. Anna owes Bill more than Clara.

(Anna owes Bill more than she owes Clara.)

b. Anna owes Bill more than Clara.

(Anna owes Bill more than Clara owes him.) 
Pollard and Smith

c. Kim read the same book as Sandy.

d. Kim read a different book than Sandy.

On our analysis, S/D sentences like (4c-d) will also be treated as remnant constructions. More specifically, the meanings of the same and different are functions that take four arguments: first, the property denoted by the common noun sister of the same or different, followed by the continuation, the associate, and the remnant.

\section{Linear Grammar}

Here we sketch just enough of the framework to (we hope) make the analyses intelligible. For more systematic expositions, see Smith 2010 or Mihalicek \& Pollard 2012.

\subsection{Generalities}

The linear grammar (LG) framework in which our analyses are couched is one of a family of categorial frameworks (others include abstract categorial grammar (de Groote 2001), lambda grammar (Muskens 2007), and hybrid categorial grammar (Kubota \& Levine 2012)) with a common intellectual heritage. From Lambek (1958) came the idea that a syntactic category can be viewed as a formula in a certain kind of propositional logic (subsequently called the Lambek calculus) and a grammar as a proof system for that logic. From Curry (1961), based on a 1948 lecture, came the idea of bifurcating syntax into tectogrammar, which has to do with the combinatorics that drive semantic composition, and phenogrammar, which is concerned with surface realization. Cresswell (1973) observed that lambda calculus could be pressed into service for doing syntax as well as semantics. Finally, Oehrle (1994) showed that, by using lambda calculus to make the phenogrammar more expressive, the tectogrammar could be correspondingly simplified.

More specifically, the phenogrammatical component of a linguistic expression is now permitted to be not just a string of words, but possibly a function over such strings. As Oehrle (1994) showed (and as we illustrate below), it now becomes possible to implement Montague's 'quantifying in' as function application in the phenogrammar. This turns out to be an important technical tool whose application extends beyond the 'lowering' of quantified NPs, and will play a key role in our analyses.

On the tectogrammatical side, the Lambek calculus can be replaced by the simpler linear logic ${ }^{4}$ which has only one logical connective (linear implication,

4 To be more precise, the tectogrammatical logic is the implicative fragment of intuitionistic linear propositional logic. 
'The same', phrasal comparatives and superlatives

written $\multimap$ ) and only two inference rules, modus ponens (MP) and hypothetical proof (HP) described below. These can be seen as roughly corresponding, respectively, to the rules Merge and Move in MGG.

\subsection{LG Technicalities}

For semanticists, the easiest way to understand LG is as a streamlined form of Montague grammar. Linguistic expressions are represented as ordered triples, written in the form

$$
\phi ; \tau ; \sigma
$$

where $\phi$, the phenogrammatical component (or simply pheno) is a lambda term; $\tau$, the tectogrammatical component (or simply tecto), is a linear logic formula; and $\sigma$, the semantic component, is another lambda term. The tecto formula can be a basic formula (such as S, NP, or N), or a complex formula recusively built from basic formulas using the $\multimap$ connective, such as NP $\multimap \mathrm{S}$ (which includes the expressions traditionally referred to as VPs), or $\mathrm{N} \multimap(\mathrm{NP} \multimap \mathrm{S}) \multimap \mathrm{S}$ (for determiners such as every). ${ }^{5}$

It is important to be aware that the pheno term and the semantic term belong to different lambda calculi (or, more precisely, different higher-order logics), with different constants and different basic types. The semantic calculus is of the familiar kind, with the usual semantic constants and basic types. But in the pheno calculus, the only basic type is s (string) and there are constants of type s for strings of length one that are the pheno components of lexical entries, e.g. anna in (6) below. Additionally, there is a constant $\mathbf{e}$ of type $\mathrm{s}$ for the null string, and a constant $\cdot$ of type $\mathrm{s} \rightarrow \mathrm{s} \rightarrow \mathrm{s}$ for string concatenation.

Placement of the natural-deduction 'turnstile' symbol $\vdash$ in front of a triple, e.g.

$$
\begin{aligned}
& \text { A Nonlogical Axiom (Lexical Entry) } \\
& \vdash \text { anna; NP; a }
\end{aligned}
$$

indicates that it is one of the signs generated by the proof system, that is, as explained below, either an axiom or else a theorem provable from the axioms via inference rules. For LG triples, being a sign is the counterpart of being 'well-formed' in other grammar frameworks.

LG analyses - the counterpart of Montague's analysis trees-are natural-deduction proof trees, in which the leaves (at the top of the tree) are labelled by axioms, and the other nodes (including the root, which is at the bottom) are licensed by instances

5 The connective $\multimap$ associates to the right, so that, e.g. $A \multimap B \multimap C$ abbreviates $A \multimap(B \multimap C)$, not $(A \multimap B) \multimap C$. 
of the inference rules MP and HP described below. ${ }^{6}$ Axioms are of two kinds: nonlogical axioms, which are lexical entries, such as (6) above; and logical axioms, which correspond roughly to traces in MGG (and we will use the terms 'trace' and 'logical axiom' interchangeably).

The fact that a trace is as-yet 'undischarged' is indicated to the left of the turnstile. For example, a trace of tectogrammatical type NP has the form:

\section{A Logical Axiom (Trace)} $s ; \mathrm{NP} ; x \vdash s ; \mathrm{NP} ; x$

Note that the pheno and semantic terms of a trace are just variables (in this case, of types s and e (entity) respectively). More generally, an expression containing $n$ undischarged traces will have $n$ triples with variable terms (all with distinct variables) to the left of the turnstile. Such triples are called hypotheses, and the collection of hypotheses to the left of the turnstyle is called a (proof-theoretic) context. The hypotheses in the context are unordered; that is, they form a finite set, not a list.

In LG linguistic analyses (proof trees), the nodes are labelled by sequents, assertions of the form

$$
\Gamma \vdash A
$$

where $\Gamma$ is a context and $A$ is a pheno-tecto-semantics triple, called the statement of the sequent. The intuitive content of such a sequent is that the grammar licenses a sign $A$ containing undischarged traces as specified in $\Gamma$. In particular, axioms, which label the leaves of proof trees, are sequents; for lexical entries, the context is null, while a trace has a single hypothesis, which is identical to its statement.

The non-leaf nodes of a proof tree are licensed by (inference) rules, which are essentially conditional assertions with a conjunction of sequents (called the premisses) as the antecedent and another sequent (called the conclusion) as the consequent. In linguistic terms, these rules can be thought of as telling us what options are available for constructing a new (mother) expression given that certain (daughter) expressions have already been constructed. Rules are written with the premisses above a horizontal line and the conclusion below it, as in (8) below. A rule licenses a (non-leaf) node in a proof tree if and only if the sequent labelling that node is the rule's conclusion and the sequents labelling the node's daughters are the rule's premisses. LG has just two schemas of rules, MP and HP, which are named after the two rules of linear logic (of which they are elaborated versions). Essentially, this means that the only options are to either (i) apply a functor expression to its argument, or (ii) bind a trace in an expression.

6 Logicians' proof trees differ cosmetically from linguists' trees in that daughters are above, not below, their mothers. 
'The same', phrasal comparatives and superlatives

In the definitions of MP and HP in (8) below and thenceforth, we adhere to the following notational conventions. Capital Greek letters are used as metavariables that range over contexts, and capital italic letters are used as metavariables that range over formulas. To conserve horizontal space, we use computer-science notational conventions for lambda terms rather than linguistic ones. Thus, we write $(f a)$ rather than $f(a)$. Outermost parentheses are usually omitted, and application is left-associative, so that $f a b$ abbreviates $((f a) b)$, which we might otherwise have written as $[f(a)](b)$. Additionally, we abbreviate successive lambda abstractions, e.g. $\lambda_{x y} . a$ for $\lambda_{x} \cdot \lambda_{y} \cdot a$.

Modus Ponens (MP)

$$
\frac{\Gamma \vdash f ; B \multimap E ; g \quad \Delta \vdash a ; B ; c}{\Gamma, \Delta \vdash f a ; E ; g c}
$$

Hypothetical Proof (HP)

$$
\frac{\Gamma, x ; B ; z \vdash d ; E ; f}{\Gamma \vdash \lambda_{x} . d ; B \multimap E ; \lambda_{z} . f}
$$

The essence of MP is that something of tecto type $B \multimap E$ can be applied to something of tecto type $B$ to yield something of type $E$ as long as the functional terms $f$ and $g$ are of the right type to be applied to the terms $a$ and $c$ respectively. Thus MP is associated with function application in the pheno and the semantics. Significantly, it is possible for both premisses to have contexts, in which case the context of the conclusion is the union of the contexts of the premisses. In linguistic analyses, this will have the consequence that a hypothesis is always propagated through the proof tree starting from the trace in whose context it is introduced, until it is discharged by an instance of HP.

In instances of HP, there is only one premiss, but it must have a nonempty context (so, at least one hypothesis). In the conclusion of HP, one of the hypotheses has been removed ('discharged'). In the tecto of the statement of the conclusion, an implication is introduced, from the tecto type of the discharged hypothesis to the tecto type of the premiss' statement. In the pheno and semantics of the conclusion, the pheno and semantic variables of the discharged hypothesis are abstracted on. Thus HP corresponds to lambda abstraction in the pheno and semantics. HP plays a role in LG and related frameworks analogous to that played by the binding of a trace by an empty operator in MGG.

To illustrate the workings of these schemas, we need the following lexical entries (here 'QP' abbreviates $(\mathrm{NP} \multimap \mathrm{S}) \multimap \mathrm{S}$, the tecto type of quantified noun phrases; and Deg is the tecto type of degree expressions): 
(9)

$$
\begin{aligned}
& \vdash \text { bill; NP; b } \\
& \vdash \text { clara; NP; } \\
& \vdash \text { two } \cdot \text { bucks; Deg; } \$ 2 \\
& \vdash \lambda_{s} . s \cdot \text { yelled; NP } \multimap \mathrm{S} ; \text { yell } \\
& \vdash \lambda_{s t} \cdot t \cdot \text { read } \cdot s ; \mathrm{NP} \multimap \mathrm{NP} \multimap \mathrm{S} \text {; read } \\
& \vdash \lambda_{s t u} \cdot u \cdot \text { owes } \cdot s \cdot t ; \mathrm{NP} \multimap \mathrm{Deg} \multimap \mathrm{NP} \multimap \mathrm{S} ; \text { owe } \\
& \vdash \text { book; } ; \text { book } \\
& \vdash \text { kid; } ; \text { kid } \\
& \vdash \lambda_{s f} \cdot f(\text { every } \cdot s) ; \mathrm{N} \multimap \mathrm{QP} ; \lambda_{P Q} \cdot \forall_{x} \cdot(P x) \rightarrow(Q x) \\
& \vdash \lambda_{s f} \cdot f(\text { some } \cdot s) ; \mathrm{N} \multimap \mathrm{QP} ; \lambda_{P Q} \cdot \exists_{x} .(P x) \wedge(Q x)
\end{aligned}
$$

In a simple example like (10), we already see a pheno term that is not a string: the pheno of the intransitive verb yelled is a function that takes a string as its argument and returns the result of concatenating that string to the length-one string that is the phonological word yelled. Note that at each step of the proof, both pheno and semantic lambda terms of the current conclusion are simplified according to the usual rules of lambda conversion.

$$
\frac{\vdash \lambda_{s} . s \cdot \text { yelled; NP } \multimap \mathrm{S} \text {; yell } \vdash \text { bill; NP; b }}{\vdash \text { bill } \cdot \text { yelled; } \mathrm{S} \text {; yell b }}
$$

The next example is slightly more complicated because the verb takes more arguments: an NP object, an additional degree complement, and an NP subject. In order to avoid increasingly smaller fonts, we will often replace proofs like (11) with multi-part proofs like (12), where we label certain subtrees and then use the labels in place of the subtrees in other trees.

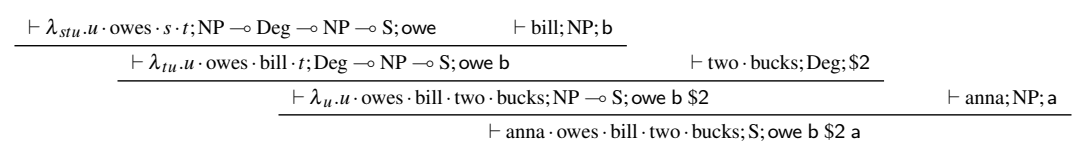

$$
\frac{\vdash \lambda_{s t u} \cdot u \cdot \text { owes } \cdot s \cdot t ; \mathrm{NP} \multimap \operatorname{Deg} \multimap \mathrm{NP} \multimap \mathrm{S} \text {; owe } \quad \vdash \text { bill; NP; b }}{\vdash \lambda_{t u} \cdot u \cdot \text { owes } \cdot \text { bill } \cdot t ; \operatorname{Deg} \multimap \mathrm{NP} \multimap \mathrm{S} \text {; owe } \mathrm{b}}
$$


'The same', phrasal comparatives and superlatives

[1] $\quad \vdash$ two $\cdot$ bucks; Deg; $\$ 2$

$\frac{\overline{\vdash \lambda_{u} \cdot u \cdot \text { owes } \cdot \text { bill } \cdot \text { two } \cdot \text { bucks; NP } \multimap \mathrm{S} \text {; owe b } \$ 2} \vdash \text { anna; NP; a }}{\vdash \text { anna } \cdot \text { owes } \cdot \text { bill } \cdot \text { two } \cdot \text { bucks; } \text {; owe b } \$ 2 \text { a }}$

The following proof trees show how QPs are formed:

[2]

$$
\frac{\vdash \lambda_{s f} . f(\text { some } \cdot s) ; \mathrm{N} \multimap \mathrm{QP} ; \lambda_{P Q} \cdot \exists_{x} \cdot(P x) \wedge(Q x) \quad \vdash \text { book; } \text {; book }}{\vdash \lambda_{f} \cdot f(\text { some } \cdot \text { book }) ; \mathrm{QP} ; \lambda_{Q} \cdot \exists_{x} .(\text { book } x) \wedge(Q x)}
$$

[3]

$$
\frac{\vdash \lambda_{s f} . f(\text { every } \cdot s) ; \mathrm{N} \multimap \mathrm{QP} ; \lambda_{P Q} \cdot \forall_{x} \cdot(P x) \rightarrow(Q x) \quad \vdash \text { kid; } ; \text {; kid }}{\vdash \lambda_{f} \cdot f(\text { every } \cdot \operatorname{kid}) ; \mathrm{QP} ; \lambda_{Q} \cdot \forall_{x} \cdot(\operatorname{kid} x) \rightarrow(Q x)}
$$

Note that the pheno of a QP is not a string, but rather a function that maps functions (from strings to strings) to strings.

The proof tree in (14) shows how to scope a subject QP outside its 'VP' (i.e. $\mathrm{NP} \multimap \mathrm{S}$ ) sister.

$$
\frac{[3] \quad \vdash \lambda_{s} . s \cdot \text { yelled; } \mathrm{NP} \multimap \mathrm{S} \text {; yell }}{\vdash \text { every } \cdot \text { kid } \cdot \text { yelled; } \mathrm{S} ; \forall_{x} .(\text { kid } x) \rightarrow(\text { yell } x)}
$$

So far, all our analyses have used only lexical entries and MP, but not traces or HP. The proof tree in (15) below illustrate Oehrle's technique of simulating Montague's quantifying-in by function application in the pheno component.

\section{Analyzing Crossed Scope $(\exists \forall)$}

[4]

$$
\frac{\vdash \lambda_{s t} . t \cdot \operatorname{read} \cdot s ; \mathrm{NP} \multimap \mathrm{NP} \multimap \mathrm{S} ; \text { read } \quad s ; \mathrm{NP} ; x \vdash s ; \mathrm{NP} ; x}{s ; \mathrm{NP} ; x \vdash \lambda_{t} . t \cdot \operatorname{read} \cdot s ; \mathrm{NP} \multimap \mathrm{S} ; \operatorname{read} x}
$$

$[5]$

[2] [4]

$$
\begin{aligned}
& s ; \mathrm{NP} ; x \vdash \text { every } \cdot \mathrm{kid} \cdot \operatorname{read} \cdot s ; \mathrm{S} ; \forall_{y} .(\operatorname{kid} y) \rightarrow(\operatorname{read} x \text { y) } \\
& \text { [3] } \quad-\lambda_{s} \text {. every } \cdot \operatorname{kid} \cdot \operatorname{read} \cdot s ; \mathrm{NP} \multimap \mathrm{S} ; \lambda_{x} \cdot \forall_{y} .(\operatorname{kid} y) \rightarrow(\operatorname{read} x y) \\
& \vdash \text { every } \cdot \operatorname{kid} \cdot \operatorname{read} \cdot \operatorname{some} \cdot \operatorname{book} ; \mathrm{S} ; \exists_{x} \cdot(\operatorname{book} x) \wedge \forall_{y} .(\operatorname{kid} y) \rightarrow(\operatorname{read} x y)
\end{aligned}
$$


Note that a trace is introduced in the position to be occupied by the wide-scoping existential QP. Essentially, in [4], read takes a trace argument to produce something of the right type to be an argument of every. Subsequently, that argument is abstracted on in [5] via HP. Finally, in the last step, the existential QP is 'lowered' into the trace position. $^{7}$

In the following section, we use all this independently motivated logical technology to analyze our target constructions: internal readings of superlatives, remnant comparatives, and both internal and remnant the same constructions. As we'll show, nothing further is required beyond the lexical entries for more, most, than, as, and same along with their meaning postulates and definitions of the relations they require, where relevant.

\section{Analyses}

\subsection{Remnant Comparatives and Internal Superlatives}

Comparatives and superlatives are used to compare, maximize, and minimize degrees. To simplify the exposition, all degrees (basic semantic type d) in this paper are degrees of monetary value. The ordering on degrees is denoted by the constant $>$ (read 'exceeds').

\subsubsection{Remnant Comparatives}

In our semantic analysis of remnant comparatives, a central role is played by the more relation, which is the ternary relation defined as follows:

$$
\operatorname{more}={ }_{\text {def }} \lambda_{r x y} \cdot\left(\max \lambda_{d} . r d x\right)>\left(\max \lambda_{d} . r d y\right)
$$

Here $r$ ranges over relations between degrees and entities, which, as we'll see, arise naturally as the continuations in remnant constructions. Additionally, we require the following two schematic lexical entries, schematic because $A$ is a metavariable ranging over tecto types (syntactic categories), and the variable $c$ ranges over functions from two strings to a single, longer string (functions of this kind are the pheno counterparts of continuations).

$$
\begin{aligned}
& \vdash \lambda_{c s t} \cdot(c \text { more } s) \cdot t ;(\operatorname{Deg} \multimap A \multimap \mathrm{S}) \multimap A \multimap \operatorname{Th}[A] \multimap \mathrm{S} \text {; more } \\
& \vdash \lambda_{s} \text {.than } \cdot s ; A \multimap \operatorname{Th}[A] ; \lambda_{x} . x
\end{aligned}
$$

By instantiating the metavariable $A$ as NP, we can analyze than-phrases such as (18):

7 Lowering corresponds roughly to covert movement (e.g. QR) in MGG. 
'The same', phrasal comparatives and superlatives

$$
\frac{\vdash \lambda_{s} \cdot \operatorname{than} \cdot s ; \mathrm{NP} \multimap \mathrm{Th}[\mathrm{NP}] ; \lambda_{x} \cdot x \quad \vdash \text { clara;NP;c }}{\vdash \text { than } \cdot \operatorname{clara} ; \mathrm{Th}[\mathrm{NP}] ; \mathrm{c}}
$$

But most of the action is in the schema for more. In the pheno, the three arguments correspond to the continuation (a function from two strings to a string), the associate (a string), and the than-phrase (a string). Essentially, this says that, as far as surface form is concerned, more and the associate are successively 'lowered' into the continuation, while the than-phrase is extraposed. ${ }^{8}$ Tectogrammatically, more takes three arguments: the sentence with two holes (one for a degree expression and one for the associate), the associate, and the than-phrase. The three occurrences of $A$ capture the category matching between the hole for the associate, the associate itself, and the remnant (the complement of than).

A typical remnant comparative is analyzed as follows. The analysis of the continuation is given first in (19), and then in (20), more is successively applied to the continuation, the associate, and the than-phrase. Here [a], [b], [m], and [o] are the lexical entries for Anna, Bill, more and owes, respectively.

\begin{tabular}{|c|c|c|}
\hline [o] & $s ; \mathrm{NP} ; x \vdash s ; \mathrm{NP} ; x$ & \\
\hline$s ; \mathrm{NP} ; x \vdash \lambda_{t u} \cdot u$ & es $\cdot s \cdot t$; Deg $\multimap \mathrm{NP} \multimap \mathrm{S}$; owe $x$ & $t$; Deg; $d \vdash t$; Deg; $d$ \\
\hline
\end{tabular}

[8]

$\frac{[7] \quad[a]}{\frac{s ; \mathrm{NP} ; x, t ; \operatorname{Deg} ; d \vdash \text { anna } \cdot \text { owes } \cdot s \cdot t ; \mathrm{S} ; \text { owe } x d \text { a }}{t \text { Deg; } d \vdash \lambda_{s} \text {.anna } \cdot \text { owes } \cdot s \cdot t ; \mathrm{NP} \multimap \mathrm{S} ; \lambda_{x} \text {.owe } x d \text { a }}}$

Note that in [7] we see our first example with more than one hypothesis in the context. In [8], the NP is discharged first, followed by the Deg. The only thing that limits the possibility of the Deg being discharged first is the fact that the Deg $\multimap S$ type that would result is not of the right type to combine with the other lexical items in the sentence we are accounting for here.

8 Successive lowering corresponds roughly to 'tucking in' (Richards 1997; Bhatt \& Takahashi 2007) in MGG, while extraposition in the present sense corresponds to 'late merge' (Bhatt \& Pancheva 2004). 
(20)

[9]

$$
\begin{aligned}
& \text { [m] [8] } \\
& \vdash \lambda_{s t} \text {. anna } \cdot \text { owes } \cdot s \cdot \text { more } \cdot t ; \mathrm{NP} \multimap \mathrm{Th}[\mathrm{NP}] \multimap \mathrm{S} \text {; more }\left(\lambda_{d x} \text {. owe } x d\right. \text { a) } \\
& \frac{[9] \quad[b]}{\frac{\vdash \lambda_{t} \text {.anna } \cdot \text { owes } \cdot \text { bill } \cdot \text { more } \cdot t ; \operatorname{Th}[\mathrm{NP}] \multimap \mathrm{S} ; \text { more }\left(\lambda_{d x} \text {.owe } x d \text { a }\right) \mathrm{b}}{\vdash \text { anna } \cdot \text { owes } \cdot \text { bill } \cdot \text { more } \cdot \text { than } \cdot \text { clara } \text { S } ; \text { more }\left(\lambda_{d x} \text {.owe } x d \text { a }\right) \text { b c }}}
\end{aligned}
$$

Finally, given the definition of more, the semantics of the conclusion reduces to:

$$
\left(\max \left(\lambda_{d} \text {.owe b } d \text { a }\right)\right)>\left(\max \left(\lambda_{d} \text {.owe c } d \text { a }\right)\right)
$$

\subsubsection{Internal Superlatives}

Turning now to most, the most relation takes two arguments. The first is a binary relation between degrees and entities, and the second is an entity. Most returns true if the entity is the only entity to have the highest degree of all entities with respect to the binary relation. We call this entity the unique maximizer, and the most relation is thus defined in terms of the unique maximizer relation um, a binary relation between entities and functions from entities to degrees. The definitions are as follows:

$$
\begin{aligned}
& \mathbf{u m}={ }_{\mathrm{def}} \lambda_{x f} \cdot \forall_{y} \cdot(y \neq x) \rightarrow((f y)<(f x)) \\
& \mathbf{m o s t}={ }_{\operatorname{def}} \lambda_{r x} \cdot \mathbf{u m} x\left(\lambda_{z} \cdot \mathbf{m a x}\left(\lambda_{d} . r d z\right)\right)
\end{aligned}
$$

To get a sense of the embedded parenthetical in the definition of most, remember that $r$ is the type of the continuation and so, in the example to come, the whole embedded parenthetical will be the amount that $z$ owes Anna. In addition to the meaning postulate, we require the following lexical entry schema:

$$
\vdash \lambda_{c s} . c \text { (the } \cdot \text { most) } s ;(\text { Deg } \multimap A \multimap \mathrm{S}) \multimap A \multimap \mathrm{S} \text {; most }
$$

In the pheno, the two arguments correspond to the continuation (a function from two strings to a string, just as in the analysis of comparatives) and the associate (a string). Essentially, this says that, as far as surface form is concerned, the most and the associate are both 'lowered' into the continuation. Tectogrammatically, the most takes two arguments: the sentence with two holes (one for a degree expression and one for the associate, again just as in the analysis of comparatives) and the associate.

Then a typical internal superlative is analyzed as follows: 
'The same', phrasal comparatives and superlatives

$$
\frac{\frac{\left.\lambda_{c s} . c \text { (the } \cdot \text { most }\right) s ;(\text { Deg } \multimap A \multimap \mathrm{S}) \multimap A \multimap \mathrm{S} \text {; most } \quad[8]}{\vdash \lambda_{s} \cdot \text { anna } \cdot \text { owes } \cdot s \cdot \text { the } \cdot \operatorname{most} ; \mathrm{NP} \multimap \mathrm{S} ; \operatorname{most}\left(\lambda_{d x} \text {.owe } x d \text { a }\right) \quad[\mathrm{b}]}}{\vdash \text { anna } \cdot \text { owes } \cdot \text { bill } \cdot \text { the } \cdot \operatorname{most} ; \mathrm{S} ; \operatorname{most}\left(\lambda_{d x} \text {.owe } x d \text { a }\right) \mathrm{b}}
$$

As in the comparative example, the metavariable $A$ is instantiated as NP. The two proof steps correspond to the successive lowering into the continuation ([8], the same one as in the comparative example) of, first, the superlative itself, and then the associate Bill. ${ }^{9}$ Finally, given the definitions of most and um, the semantics of the conclusion reduces to

$$
\forall y .(y \neq b) \rightarrow\left(\left(\max \left(\lambda_{d} \text {.owe } y d a\right)\right)<\left(\max \left(\lambda_{d} \text {.owe b } d \text { a }\right)\right)\right)
$$

\subsection{The Same Constructions}

Not surprisingly, the same relation is crucial to our analysis of sentences containing the same. The same relation is a ternary relation between a set of entities (the meaning of the common noun sister of the same), a binary relation (the continuation), and another set (the things being compared, which, for purposes of expository simplicity, we also assume to be entities). The difference between the remnant same construction and the internal same construction is that, in the former, the set of things being compared is a doubleton set, whose members are the meaning of the associate and the meaning of the remnant; whereas, in the latter, it is the set of atoms of the plural denoted by the associate.

In order to define the same relation, we first define some semantic terms for talking about (binary) relations (type $\mathrm{e} \rightarrow \mathrm{e} \rightarrow \mathrm{t}$ ). ${ }^{10}$ When we speak of a relation, what we really mean is not a set of ordered pairs, but rather the curried version of the characteristic function of such a set. Because such relations often arise as the meanings of transitive verbs, and because the grammar is written in such a way that the verb usually combines with the object before the subject, the argument that would normally be the first component of an ordered pair is actually the second argument of the curried function, and conversely. Concomitantly, a relation $r$ is called functional provided, for all $x, y, z$, if $r x z$ and $r y z$, then $x=y$; that is, the first and second argument correspond, counterintuitively, to the range and domain of the function, respectively; and $r$ is called constant provided its range is a singleton set. The higher-order formalizations of these terms are as follows:

9 Below, we'll see that internal S/D constructions are also analyzed via successive lowering, corresponding to Barker's (2007) 'parasitic scope'. However, on our account, lowered associates are not scope-taking, not even in internal S/D constructions.

10 The analysis generalizes to binary relations between two things of the same type, not necessarily e. 


\section{Definitions for Binary Relations}

$$
\begin{aligned}
& \operatorname{dom}={ }_{\operatorname{def}} \lambda_{s x} \cdot \exists_{y} . s \text { y } x \\
& \text { ran }={ }_{\text {def }} \lambda_{s y} \cdot \exists_{x} . s \text { y } x \\
& \text { funct }={ }_{\operatorname{def}} \lambda_{s} \cdot \forall_{x y z} \cdot((s x z) \wedge(s y z)) \rightarrow(x=y) \\
& \text { const }={ }_{\text {def }} \lambda_{s} \cdot \exists_{y} .(\operatorname{ran} s)=\lambda_{x} \cdot x=y
\end{aligned}
$$

Then the same relation is defined as follows:

$$
\begin{aligned}
& \text { same }={ }_{\operatorname{def}} \lambda_{P r Q} \cdot \exists_{s} .(\text { funct } s) \wedge(\text { const } s) \wedge((\text { dom } s)=Q) \wedge \\
& \forall_{x y} .(\text { s } y x) \rightarrow((P y) \wedge(r y x))
\end{aligned}
$$

\subsubsection{Remnant The Same Constructions}

The entire analysis of remnant the same constructions resides in the following lexical schemas:

$$
\begin{aligned}
& \vdash \lambda_{\text {sctu }} \cdot(c(\text { the } \cdot \text { same } \cdot s) t) \cdot u ; \mathrm{N} \multimap(\mathrm{NP} \multimap A \multimap \mathrm{S}) \multimap A \multimap \mathrm{As}[A] \multimap \mathrm{S} ; \\
& \lambda_{\text {Prxy }} \text {.same } P r\left(\lambda_{z} \cdot(z=x) \vee(z=y)\right) \\
& \vdash \lambda_{s} \text {.as } \cdot s ; A \multimap \operatorname{As}[A] ; \lambda_{x} . x
\end{aligned}
$$

Semantically, the same and the relation same have the same first two arguments: a property (e.g. book) and a two-hole continuation (in our next example, simply read, with holes corresponding to the subject and the object). The third argument of same corresponds to the set of entities that are similar in some respect. The last two arguments of the same correspond, respectively, to the associate and the remnant, and the body of the lambda-abstract ensures that it is the set of just these two entities which are similar. From these lexical schemas, then, we can construct remnant-the same-NPs and as-phrases as follows (here [s] is the lexical entry for the same with the metavariable $A$ instantiated as NP):

$$
\begin{gathered}
{[\mathrm{s}] \quad \vdash \text { book; } \text {; book }} \\
\left.\qquad \vdash \lambda_{\text {ctu }} \cdot(c \text { (the } \cdot \text { same } \cdot \text { book }) t\right) \cdot u ;(\mathrm{NP} \multimap \mathrm{NP} \multimap S) \multimap \mathrm{NP} \multimap \mathrm{As}[\mathrm{NP}] \multimap \mathrm{S} ; \\
\lambda_{r x y} \cdot \text { same book } r\left(\lambda_{z} \cdot((z=x) \vee(z=y))\right)
\end{gathered}
$$

$$
\frac{\vdash \lambda_{s} \text {.as } \cdot s ; \mathrm{NP} \multimap \mathrm{As}[\mathrm{NP}] ; \lambda_{x} \cdot x \quad \vdash \text { bill; NP; b }}{\vdash \text { as } \cdot \text { bill; As }[\mathrm{NP}] ; \mathrm{b}}
$$


'The same', phrasal comparatives and superlatives

It is instructive to compare the conclusion of [10] with that of the lexical entry for remnant more in (17), since one can see at a glance the similarities (and also the differences) between the two remnant constructions.

Then a typical remnant the same-sentence, Anna read the same book as Bill, is analyzed as follows: (here [r] is the lexical entry for read in (9)):

$\frac{[10] \quad[\mathrm{r}]}{\frac{\mathrm{RTSB}}{\mathrm{ARTSB}}[\mathrm{a}]}$
$\frac{[11]}{}$
$\quad$ anna $\cdot \mathrm{read} \cdot$ the $\cdot \mathrm{same} \cdot \mathrm{book} \cdot \mathrm{as} \cdot \mathrm{bill} ; \mathrm{S} ;$
same book read $\lambda_{z} \cdot((z=\mathrm{a}) \vee(z=\mathrm{b}))$

Here the computation of the intermediate results of successively applying the same book to the continuation read, the associate Anna, and the as-phrase as Bill, are omitted for reasons of space, as is the verification that the semantics of the conclusion same book read a b aserts the existence of a constant function that maps each of Anna and Bill to a book that $\mathrm{s} / \mathrm{he}$ read. (The analysis of the comparative example (20) can be used as a model; the only rule used here is MP.)

\subsubsection{Internal The Same Constructions}

Finally, we analyze the internal the same construction, which is the construction analyzed by Barker 2007 under the rubric of 'parasitic scope'. As might be expected, the analysis is driven by another lexical schema:

$$
\begin{aligned}
& \vdash \lambda_{s c t} . c(\text { the } \cdot \text { same } \cdot s) t ; \mathrm{N} \multimap(\mathrm{NP} \multimap A \multimap \mathrm{S}) \multimap A \multimap \mathrm{S} ; \\
& \lambda_{P r X} \text {. same } \operatorname{Pr}(\text { at } X)
\end{aligned}
$$

The variable $X$, corresponding to the third semantic argument, ranges over plurals. Also, the constant at denotes the function that maps plurals to the set of their atomic elements.

From this, we can construct a the same-NP as follows (where [s'] is the lexical entry that instantiates $A$ as NP in the preceding schema):

$$
\begin{gathered}
\frac{\left[\mathrm{s}^{\prime}\right] \quad \vdash \text { book; } \mathrm{N} \text {; book }}{\vdash \lambda_{c t} . c(\text { the } \cdot \text { same } \cdot \text { book }) t ;(\mathrm{NP} \multimap \mathrm{NP} \multimap \mathrm{S}) \multimap \mathrm{NP} \multimap \mathrm{S} ;} \\
\lambda_{r X} \text {.same book } r(\text { at } X)
\end{gathered}
$$


Comparing the tecto type of [12] with that of a quantified NP (namely, $(\mathrm{NP} \multimap \mathrm{S}) \multimap$ S) shows why all attempts to analyze internal the same-NPs as generalized quantifiers have failed: they are of the wrong type. (More specifically, GQs scope over onehole continuations, not two-hole continuations.) It's instructive in a different way to compare [12] with the very similar lexical schema for internal more: in both cases, the expression in question lowers first itself, and then the associate, into the two-holed continuation.

Finally, (34) is our analysis of a typical internal-the same sentence, Anna and Bill read the same book. This analysis presupposes some analysis or other of coordinate NPs according to which Anna and Bill is interpreted as $a+b$, the plural whose atomic elements are Anna and Bill. It has the same meaning as the remnant the same example in (31), since $a+b=\lambda_{z} \cdot((z=a) \vee(z=b))$.

$$
\begin{aligned}
& \text { [12] [r] } \\
& \vdash \lambda_{t} \cdot t \cdot \mathrm{read} \cdot \text { the } \cdot \text { same } \cdot \text { book; } \mathrm{NP} \multimap \mathrm{S} \text {; } \\
& \lambda_{X} \text {.same book read }(\text { at } X) \\
& \text { [13] } \vdash \text { anna } \cdot \text { and } \cdot \text { bill; NP; } a+b \\
& \vdash \text { anna } \cdot \text { and } \cdot \text { bill } \cdot \text { read } \cdot \text { the } \cdot \text { same } \cdot \text { book; S; same book read } a+b
\end{aligned}
$$

\section{Conclusion}

The analysis of internal the same constructions above incorporates Barker's (2007) key insight — which also informs all the other analyses in this paper-that some expressions take as an argument a two-holed continuation, one of whose holes is for the expression itself. But our analysis extends to a wider range of phenomena, including remnant constructions and internal superlatives, thereby capturing the new generalization that the second hole is for an associate, which need not itself be a scope-taking element. Moreover, our analysis is simpler than Barker's in numerous respects: no choice functions, only one syntactic mode of combination, and no structural rules for the grammar logic. At the same time, building on a long tradition of logic-based grammatical analysis initiated by Haskell Curry in the late 1940's, independent of the MGG tradition, we provide simple, straightforward reconstructions of a wide range of MGG notions - such as covert movement/QR, ellipsis, tucking in, and late merge — based on the most elementary proof-theoretic concepts.

\section{References}

Barker, Chris. 2007. Parasitic scope. Linguistics and Philosophy 30. 407 - 444. 
'The same', phrasal comparatives and superlatives

Bhatt, Rajesh \& Roumanya Pancheva. 2004. Late merger of degree clauses. Linguistic Inquiry 35. 1 - 45.

Bhatt, Rajesh \& Shoichi Takahashi. 2007. Direct comparisons: resurrecting the direct analysis of phrasal comparatives. In Semantics and Linguistic Theory (SALT) 17, 19 - 36. Ithaca, NY: CLC.

Cresswell, Max. 1973. Logics and Languages. London: Methuen.

Curry, Haskell. 1961. Some logical aspects of grammatical structure. In Roman O. Jakobson (ed.), Structure of Language and its Mathematical Aspects, 56 - 68. Providence, RI: American Mathematical Society.

de Groote, Philippe. 2001. Toward abstract categorial grammars. In Association for Computational Linguistics (ACL) 39, 148 - 155.

Heim, Irene. ms. Notes on comparatives and related matters. Unpublished manuscript, University of Texas at Austin.

Kennedy, Chris. 1999. Projecting the Adjective: The Syntax and Semantics of Gradability and Comparison. Outstanding Dissertations in Linguistics Series. Garland.

Kubota, Yusuke \& Robert Levine. 2012. Gapping as like-category coordination. In Logical Aspects of Computational Linguistics (LACL) 7, 135-150.

Lambek, Joachim. 1958. The mathematics of sentence structure. American Mathematical Monthly 65. $154-170$.

Mihalicek, Vedrana \& Carl Pollard. 2012. Distinguishing phenogrammar from tectogrammar simplifies the analysis of interrogatives. In Formal Grammar $(F G)$ 15 (2010), 130 - 145. Springer.

Muskens, Reinhard. 2007. Separating syntax and combinatorics in categorial grammar. Research on Language and Computation 267 - 285.

Oehrle, Richard. 1994. Term-labelled categorial type systems. Linguistics and Philosophy 17. 633-678.

Richards, Norvin. 1997. What moves where in which language? Cambridge, MA: MIT dissertation.

Smith, E. Allyn. 2010. Correlational Comparison in English. Columbus, OH: The Ohio State University dissertation.

Carl Pollard

222 Oxley Hall

The Ohio State University

Columbus OH 43210

pollard@ling.ohio-state.edu
E. Allyn Smith

University of Quebec at Montreal

Montreal (Quebec) H3S 3P8

Canada

smith.elizabeth_allyn@uqam.ca 